\title{
Estimation of the frequency of inherited germline mutations by whole exome sequencing in ethyl nitrosourea-treated and untreated gpt delta mice
}

Kenichi Masumura ${ }^{1 *}$, Naomi Toyoda-Hokaiwado ${ }^{1}$, Akiko Ukai ${ }^{1}$, Yoichi Gondo², Masamitsu Honma ${ }^{1}$ and Takehiko Nohmi ${ }^{1,3}$

\begin{abstract}
Background: Germline mutations are heritable and may cause health disadvantages in the next generation. To investigate trans-generational mutations, we treated male gpt delta mice with $N$-ethyl- $N$-nitrosourea (ENU) (85 mg/kg intraperitoneally, weekly on two occasions). The mice were mated with untreated female mice and offspring were obtained. Whole exome sequencing analyses were performed to identify de novo mutations in the offspring.

Results: At 20 weeks after the treatment, the gpt mutant frequencies in the sperm of ENU-treated mice were 21-fold higher than those in the untreated control. Liver DNA was extracted from six mice, including the father, mother, and four offspring from each family of the ENU-treated or untreated mice. In total, 12 DNA samples were subjected to whole exome sequencing analyses. We identified de novo mutations in the offspring by comparing single nucleotide variations in the parents and offspring. In the ENU-treated group, we detected 148 mutation candidates in four offspring and $123(82 \%)$ were confirmed as true mutations by Sanger sequencing. In the control group, we detected 12 candidate mutations, of which, three (25\%) were confirmed. The frequency of inherited mutations in the offspring from the ENU-treated family was $184 \times 10^{-8}$ per base, which was 17 -fold higher than that in the control family $\left(11 \times 10^{-8}\right.$ per base). The de novo mutation spectrum in the next generation exhibited characteristic ENU-induced somatic mutations, such as base substitutions at A:T bp.

Conclusions: These results suggest that direct sequencing analyses can be a useful tool for investigating inherited germline mutations and that the germ cells could be a good endpoint for evaluating germline mutations, which are transmitted to offspring as inherited mutations.
\end{abstract}

Keywords: Exome sequencing, Germline mutation, Heritable mutation, Mutation spectrum, Transgenic rodent gene mutation assay

\footnotetext{
* Correspondence: masumura@nihs.go.jp

${ }^{1}$ Division of Genetics and Mutagenesis, National Institute of Health Sciences,

1-18-1 Kamiyoga, Setagaya-ku, Tokyo 158-8501, Japan

Full list of author information is available at the end of the article
} 


\section{Background}

Gene mutations induced in germ cells may cause heritable changes, which can have adverse health effects on the next generation. Thus, analyses of germ cell mutations and risk evaluations for trans-generational mutagenesis induced by chemicals are important research subjects in genotoxicology [1]. Many genotoxicity and mutagenicity tests employ somatic cells to detect mutagens, which may have carcinogenic potential. Indeed, about 50 mutagens have been analyzed as rodent germ cell mutagens and about 40 of them were positive [2]. Mouse-specific locus tests and mouse heritable translocation tests have been used to detect transgenerational mutations [3, 4]; however, these tests are rarely performed anymore as they need considerably large numbers (hundreds to thousands) of animals. Some genotoxicity/mutagenicity tests are also applicable to germ cells, e.g., transgenic rodent (TGR) gene mutation assays are useful for detecting mutations in both somatic and male germ cells [5]. Recent advances in high-throughput sequencing techniques have extended the use of direct sequencing to mutation analyses. Thus, a combination of TGR mutation assay and nextgeneration sequencing (NGS) could be a comprehensive approach for analyzing heritable mutations. In this study, we estimated the $N$-ethyl- $N$-nitrosourea (ENU)-induced germline mutation frequency (MF) in gpt delta transgenic mice by whole exome sequencing using NGS. ENU is a known germ cell mutagen, and it has been used to generate mutant mice in large-scale chemical mutagenesis projects [6-8]. We treated male mice with ENU and mated them with untreated females at 10 weeks after the treatment. Whole exome sequencing was performed for the parents and offspring of the ENU-treated and control families, and de novo germline mutations were detected by comparing single nucleotide variants (SNVs) in the parents and offspring. We also estimated the gpt mutant frequency in sperm DNA from the ENU-treated mice. Our results showed that the frequency of inherited mutations was clearly higher in the ENU-treated group than that in the control group. The mutation spectrum determined by NGS indicated the presence of characteristic ENU-induced mutations. Thus, NGS may be a powerful approach for analyzing chemically induced transgenerational mutations, and germ cells could be a good surrogate for trans-generational mutagenesis studies.

\section{Methods}

\section{Treatment of animal}

Male and female gpt delta mice (C57BL/6 J background) $[9,10]$ were obtained from a breeding colony maintained at the National Institute of Health Sciences. The animal treatment employed in this study was approved by the Animal Care and Utilization Committee of the institute.
The experimental design was based on a protocol for mouse mutagenesis using ENU [11] and a previously reported RIKEN ENU mutagenesis project [12], with some modifications. Nine-week-old male mice were treated with ENU (85 $\mathrm{mg} / \mathrm{kg}$ body weight intraperitoneally, weekly on two occasions) (Additional file 1: Figure S1). The male mice were pre-mated with untreated female mice at 6-7 weeks after the last treatment to check for a period of infertility induced by ENU. Exposure to ENU was confirmed by their temporal sterility. Ten weeks after the last treatment, the male mice were mated with untreated female mice and F1 offspring were obtained. Control male mice were treated with phosphate/citrate buffer as vehicle and mated with untreated females without a pre-mating period. Five male mice were used in each group. After the offspring were obtained, male mice were sacrificed at 20 weeks after the last treatment (30 week-old), and their tissues were then collected and stored at $-80{ }^{\circ} \mathrm{C}$. The mated females and the offspring were also sacrificed at $30-33$ and 5 weeks old, respectively.

\section{Reporter gene mutation assay}

Genomic DNA was extracted from the liver using a RecoverEase DNA Isolation Kit (Agilent Technologies, Santa Clara, CA). Sperm DNA was extracted as described [13] with some modifications. In brief, the cauda epididymis was chopped in $1 \mathrm{~mL}$ of phosphate-buffered saline ( $\mathrm{pH} 7.4)$, filtered, and pelleted by centrifugation. The pellet was re-suspended in $1 \times$ saline sodium citrate (SSC) and $0.15 \%$ sodium dodecyl sulfate (SDS). The lysate was centrifuged and the sperm pellet was suspended in $1 \mathrm{~mL}$ of $0.2 \times \mathrm{SSC}, 1 \% \mathrm{SDS}, 1 \mathrm{M} 2$-mercaptoethanol, and $10 \mathrm{mM}$ EDTA ( $\mathrm{pH} 8.0$ ), before digesting overnight with $0.5 \mathrm{mg} / \mathrm{mL}$ proteinase $\mathrm{K}$ at $37{ }^{\circ} \mathrm{C}$. DNA was isolated by extracting four times in phenol/chloroform, ethanol precipitation, and re-suspension in TE buffer ( $\mathrm{pH}$ 8.0). For the gpt gene mutation assay, lambda EG10 transgenes were rescued by in vitro packaging reactions as phage particles using Transpack Packaging Extract (Agilent Technologies). The gpt mutation assay was performed as described previously [14]. In brief, the rescued phages were used to infect Escherichia coli strain YG6020, which expressed Cre recombinase to convert the transgene into a plasmid. The infected cells were mixed with molten soft agar and poured onto M9 agar plates containing chloramphenicol $(\mathrm{Cm})$ and 6thioguanine (6TG). The plates were incubated for 4 days at $37^{\circ} \mathrm{C}$ to select colonies that harbored the plasmid carrying the mutated gpt gene. Infected cells were also poured onto plates containing $\mathrm{Cm}$ without 6TG to determine the number of rescued plasmids. The gpt mutant frequencies were calculated by dividing the number of 6TG-resistant colonies by the number of rescued plasmids. The gpt 
mutants obtained from the sperm of ENU-treated mice were sequenced to determine the gpt mutations using an ABI3730 sequencer (Applied Biosystems by Life Technologies, Carlsbad, CA) with a sequencing primer gptA2 (5'TCTCGCGCAACCTATTTTCCC-3').

\section{High-throughput DNA sequencing analysis}

One ENU-treated family and one control family were employed in the high-throughput sequencing analyses (Fig. 1), where each family comprised six mice, i.e., parents (male and female) and offspring (two males and two females). Genomic DNA was extracted from the liver using a Wako DNA Extractor WB Kit (Wako, Osaka, Japan). Liver DNA samples from 12 mice were subjected to NGS analyses by Beckman Coulter Genomics (MA, USA). Genomic DNA was fragmented using the Covaris DNA shearing system (Covaris, Inc., MA, USA). The whole mouse exome (49.6 Mb) was captured using a SureSelect Mouse All Exon Kit (Agilent Technologies) and sequenced by a Hiseq2000 (Illumina, CA, USA) with 100-bp paired-ends. Sequenced reads were mapped onto the reference sequence using ELAND (Illumina). The reference sequence was C57BL/6 J mouse genome: NCBI Build 37, mm9 (http://www.ncbi.nlm.nih.gov/projects/ genome/assembly/grc/mouse/), and the SureSelect Mouse All Exon Kit was designed using the same reference sequence. For each animal, the SNVs were called based on comparisons with the reference sequence using Samtools.

\section{Calculation of the frequency of inherited mutations in the offspring of ENU-treated and control fathers}

The SNVs were compared in the parents and offspring, and de novo mutations were identified as follows. (1) The SNVs transmitted from the parents were excluded. (2) The SNVs found only in one offspring (not found in the other 11 mice) were considered as unique mutations and selected as candidate de novo mutations. (3) The read depth, i.e., the number of sequencing reads that covered one nucleotide position, had to exceed 40 in all three mice, i.e., father, mother, and offspring (see the Results). (4) The genotype quality (GQ) scores in NGS had to exceed 20 (=99\% accuracy) in all three mice. The frequency of inherited mutations was calculated as follows. The number of de novo mutations was divided by the number of bases in the exome with the same cut-off value, i.e., a read depth $\geq 40$ and GQ score $\geq 20$ in all three mice. The NGS data were analyzed by Genaris Omics Inc. (Kanagawa, Japan). Confirmation of the mutation candidates by Sanger sequencing was performed by TAKARA BIO Inc. (Shiga, Japan) using an ABI3730 sequencer (Applied Biosystems by Life Technologies) with custom-designed PCR primers for each mutated position in the genome of offspring.

\section{Results}

\section{ENU treatment and gpt mutant frequency}

The male mice were treated with ENU and a pre-mating period was enforced to check their temporal infertility due to ENU at weeks 6-7 after the last treatment. No pregnancies occurred during the pre-mating period. At week 10 , the mice were mated with untreated females to obtain offspring. In total, 24 F1 mice comprising 17 males and 7 females were obtained from five mated pairs. Tissue samples were collected from the paternal, maternal, and F1 mice. Exposure to ENU was confirmed by the gpt mutation assay (Fig. 2). The gpt mutant frequency in the liver of ENU-treated mice $\left(88.4 \pm 25.4 \times 10^{-6}\right)$ was 37 -fold higher than that in the control mice $\left(2.4 \pm 1.5 \times 10^{-6}\right)$. In the sperm of ENU-treated mice, the gpt mutant frequency $\left(44.4 \pm 25.9 \times 10^{-6}\right)$ was 21 -fold higher than that of the control mice $\left(2.1 \pm 1.7 \times 10^{-6}\right)$. In the offspring of ENUtreated fathers, the gpt mutant frequency in the liver $\left(3.0 \pm 3.6 \times 10^{-6}\right)$ did not differ significantly from that of the control fathers. One ENU-treated mouse (ID = 8: gpt mutant frequency $=74.1 \times 10^{-6}$ ) and one control mouse $\left(\right.$ ID $=43$ : gpt mutant frequency $\left.=1.2 \times 10^{-6}\right)$ were selected randomly, and their families with the paired female and offspring were used for the NGS analyses.

\section{Frequency of inherited mutations in ENU-treated mice} Genomic DNA samples prepared from the livers of one ENU-treated and one vehicle-treated family were subjected
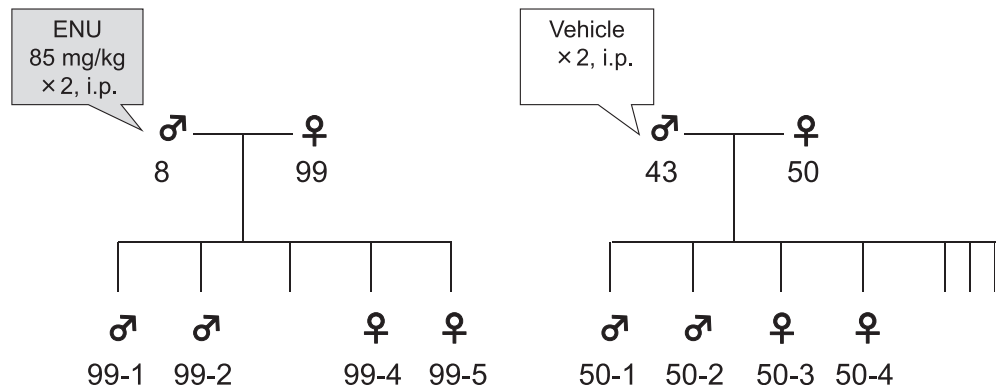

Fig. 1 Mouse families used for analyses of whole exome sequencing. For each family, six mice comprising the parents (one male and one female) and four offspring (two males and two females) were used for whole exome sequencing analyses. Each animal ID is presented 


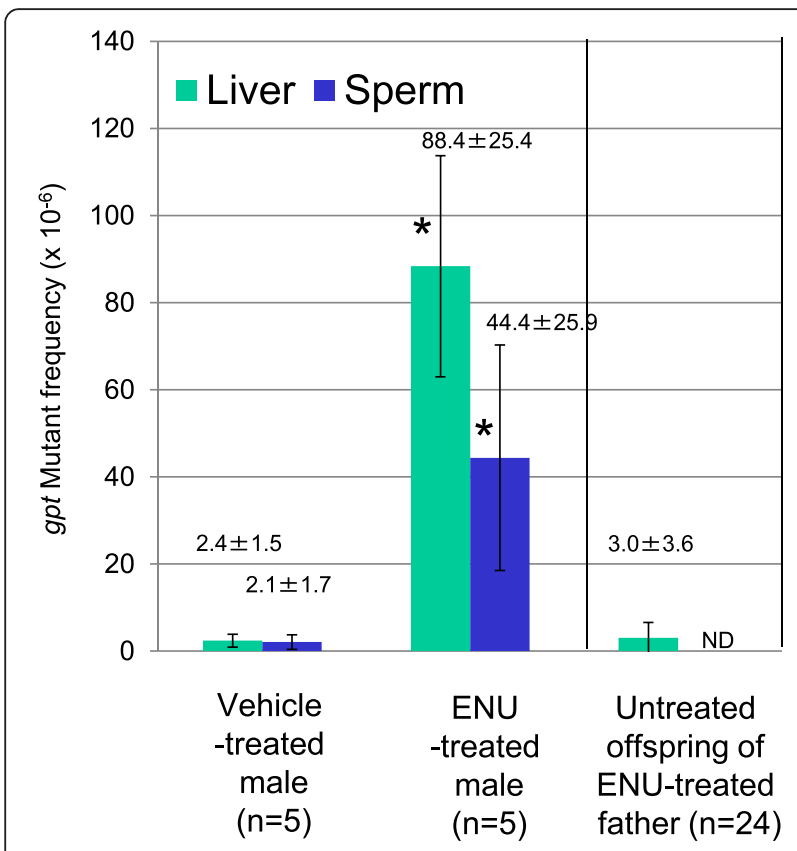

Fig. 2 The gpt mutant frequencies in the liver and sperm of the ENU-treated mice and their offspring. The gpt mutant frequencies are presented with the standard deviations. ND: not determined. Asterisks indicates significant differences vs the vehicle control ( $p<0.05$, Kruskal-Wallis test)

to whole exome sequencing (Fig. 1). Initial read data comprising 12-20 gigabases (Gb) were obtained from each animal. The sequenced reads were mapped onto the reference mouse genome sequence, where 54-66 \% of the mapped reads were mapped onto the exon region. Thus, over $5 \mathrm{~Gb}$ of the sequenced data were mapped onto the $49.6-\mathrm{Mb}$ exon region (approximately 100-fold redundancy). For $70 \%$ of the bases in the exome, the read depth exceeded
30. For each animal, SNVs and small Indels were detected based on comparisons with the reference sequence.

The SNVs were compared in the parents and offspring, and de novo mutation candidates were scored in the offspring (unique mutations only). The mutation candidates were sorted by using the read depth as a cutoff value. When a higher read depth was set, less mutation candidates were sorted (Additional file 2: Table S1). The ratio of the total number of mutation candidates in the ENU-treated group relative to that of the candidates in the control group was plotted as well as the read depth (Fig. 3). The ratio increased with the read depth and peaked when the read depth $\geq 40$, at which it was $>12$. This was the most sensitive setting for detecting ENUinduced de novo germline mutations under these experimental conditions. Therefore, a read depth $\geq 40$ in all three mice (father, mother, and one offspring) was selected as the cut-off value to calculate the frequency of inherited mutations.

In the ENU group, 148 candidate mutations were detected from four offsprings, whereas, 12 candidate mutations were detected in the control group (Table 1). Most mutation candidates (157/160) were single base substitutions and three were small indels (two single base deletions and one 2-base deletion) (Additional file 2: Table S2). These candidate mutations were examined by Sanger sequencing. In the ENU group, 83 \% (123/148) were confirmed as true mutations, whereas there were many false calls in the control group and only $25 \%(3 / 12)$ were confirmed. All were detected as heterozygous point mutations in the genomes of the offspring (Additional file 2: Table S2). The MFs of each offspring were calculated by dividing the number of mutations by the number of the bases in exome, which was calculated using the cut-off condition, i.e., read depth $\geq 40$ in all

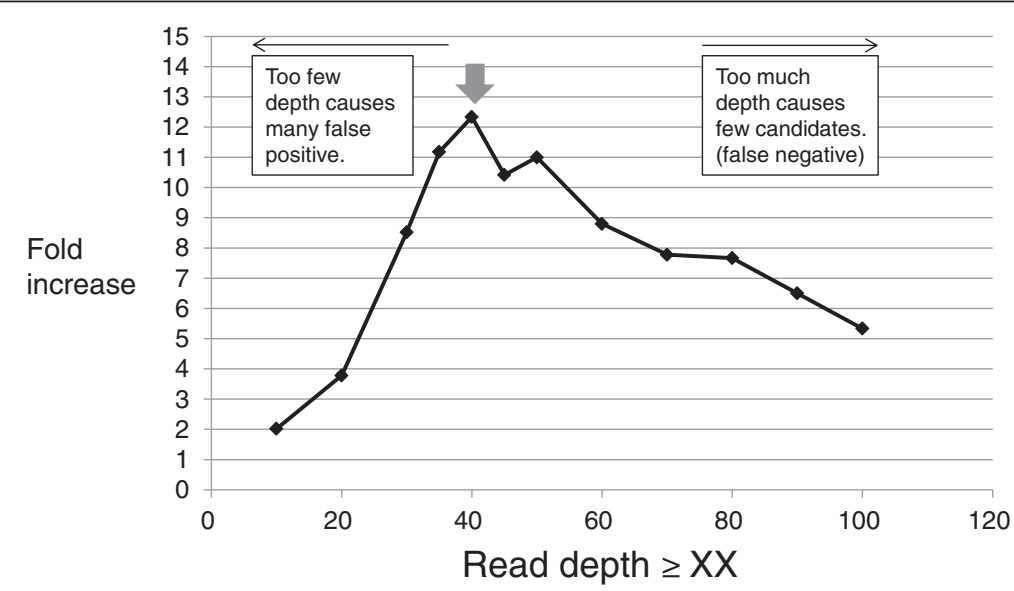

Fig. 3 Relative ratio of the number of mutations detected in the offspring of ENU-treated and vehicle-treated fathers. Ratio of the total number of de novo candidate mutations in four offspring from the ENU and control group. The thick arrow indicates the peak of the fold increase. When read depth $\geq 40$, the number of mutations in the ENU-treated group was 12-fold higher than that in the control group 
Table 1 Frequencies of inherited mutations in the offspring of ENU- or vehicle-treated father

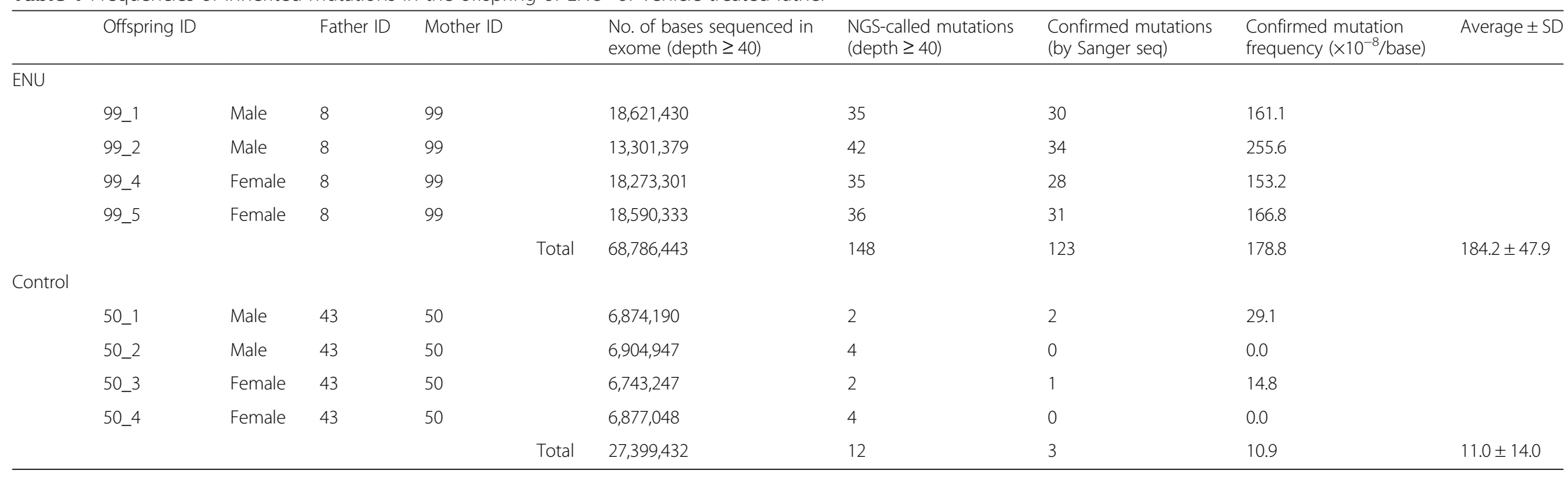


three mice (Additional file 3: Figure S2). The frequency of inherited mutations in the ENU-treated mice was $184 \pm 48 \times 10^{-8}$ bases, which was 17 -fold higher than that in the control group $\left(11 \pm 14 \times 10^{-8}\right.$ bases). In the control group, fewer bases were sorted in the exome compared with the ENU group because the NGS data quality for animal ID 43, which was the father of the control family, was lower compared with the quality for the other mice analyzed. This low quality reduced the number of mutation candidates that exceeded the cut-off value. In this study, the false positive MF was about $30 \times 10^{-8}$ bases in both the ENU and control groups (Fig. 4).

\section{Mutation spectra of inherited mutations in ENU-treated mice}

The spectrum of inherited mutations in the ENU-treated mice is shown in Table 2. Among 123 confirmed mutations, base substitutions at A:T bps were mainly observed $(90 / 123=73.2 \%)$. A:T to G:C transitions occurred in $42.3 \%$ of cases $(52 / 123)$ and A:T to T:A transversions were observed frequently $(33 / 123=26.8 \%)$. The control group had only three confirmed mutations. No mutational hotspots were observed in the ENU-induced mutations (Additional file 2: Table S2). Non-synonymous mutations in coding sequences accounted for $45 \%$ of cases $(55 / 123)$ and $9 \%$ were synonymous mutations (11/123) (Additional file 2: Table S3). Intronic mutations comprised $19 \%$ of cases (23/123). In two cases, two mutations were identified at neighboring bases in the same animal, where A to $\mathrm{G}$ at 27827556 and $\mathrm{A}$ to $\mathrm{T}$ at 27827557 in chromosome 5 of mouse ID 99_2 may be considered as AA to GT tandem mutations, while $\mathrm{T}$ to $\mathrm{C}$ at 119834044 and $\mathrm{A}$ to $\mathrm{T}$ at

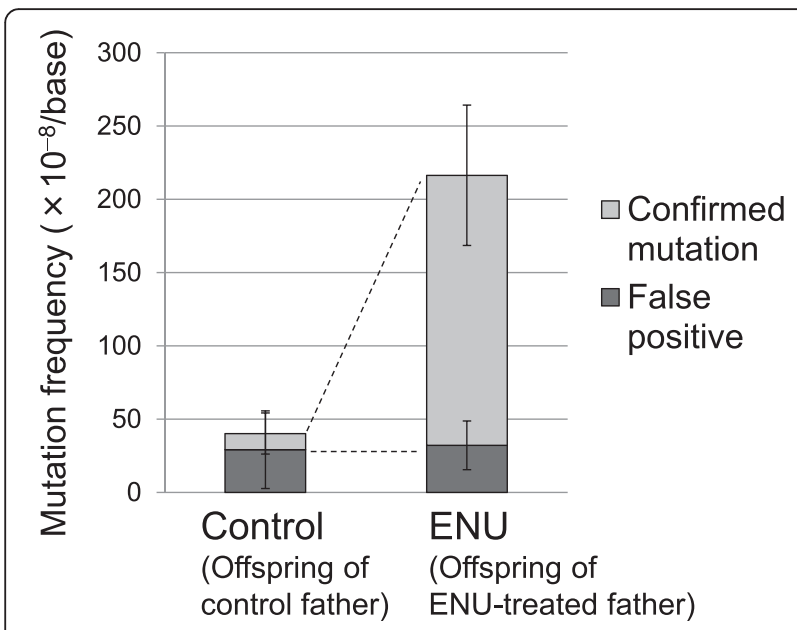

Fig. 4 Frequencies of inherited mutations in the offspring of ENU-treated or vehicle-treated fathers. Each bar represents average of 4 offspring with standard deviation. The confirmed mutation frequency was 17-fold higher in the offspring of the ENU-treated father. The frequency of false positives was about $30 \times 10^{-8}$ bases
119834046 in chromosome 4 of mouse ID 99_5 may be considered as TTA to CTT mutations.

The mutation spectrum of the gpt mutations in the sperm of ENU-treated male mice is shown in Table 2, which indicates that A:T to T:A transversions (34.7\%) and G:C to A:T transitions (22.4\%) were mainly observed.

\section{Discussion}

De novo mutations in the offspring of the ENU-treated father were identified by whole exome sequencing followed by Sanger sequencing. Theoretically, the minimum dataset required for analyzing de novo mutations is a trio (parents and one offspring). However, using such a small sample number may lead to many false positive calls. It is expected that ENU induces point mutations randomly in the genome at a frequency of $1 / \mathrm{Mbp}$ [12]. Thus, multiple identical mutations in different individuals could be treated as sequencing errors rather than independent mutations. To minimize the false mutation calls, we counted unique mutations detected in one mouse among the 12 mice used in this study as de novo candidate mutations. After simply sorting the unique candidate mutations by read depth $\geq 40$ in all three mice (father, mother, and one offspring), the number of candidate mutations in the offspring of the ENU-treated father was over 12 times higher than that in the control. Confirmation by Sanger sequencing indicated that $83 \%(123 / 148)$ of the ENUinduced de novo candidate mutations were true mutations. By contrast, only $25 \%$ (3/12) were confirmed in the control group, and there were many false calls. In this study, false positives mean that Sanger sequencing using the same DNA sample could not confirm the potential NGS mutations (Additional file 2: Table S2). Sanger sequencing may not be able to confirm the mutations in some cases. For example, mismatches or multiple targets of PCR primers may cause failed PCR amplification. In another example, if multiple copies of identical sequence are distributed in the genome, PCR may not preferentially amplify the mutated locus and Sanger sequencing cannot detect a signal of the mutated base. However, there is no perfect explanation for discrepancy between NGS and Sanger sequencing. SNVs detected by NGS may contain some errors even if they have sufficient read depth.

Interestingly, the false positive MFs, i.e., approximately $30 \times 10^{-8}$ bases, was similar in both the control and ENU-treated groups (Fig. 4), which may indicate the background level of false positives under this experimental condition. To reduce false positives, it may be necessary to employ more animals, deeper sequencing, longer target sequences, and to improve the SNV detection methods. However, the false-negative rate was not determined in this study. The candidate mutations were sorted to maximize the sensitivity of the method, i.e., according to the ratio of a number of candidate mutations 
Table 2 Mutation spectra in the offspring and somatic tissues of ENU-treated mice

\begin{tabular}{|c|c|c|c|c|c|c|c|c|c|c|c|c|c|c|}
\hline \multirow[b]{3}{*}{$\begin{array}{l}\text { Target } \\
\text { sequences }\end{array}$} & \multirow{2}{*}{\multicolumn{2}{|c|}{$\begin{array}{l}\text { Control } \\
\text { NGS (this } \\
\text { study) }\end{array}$}} & \multicolumn{12}{|l|}{ ENU } \\
\hline & & & \multicolumn{2}{|l|}{ NGS (this study) } & \multicolumn{2}{|l|}{$\begin{array}{l}\text { Genome-wide } \\
\text { screening }\end{array}$} & \multicolumn{6}{|c|}{ Transgenic reporter gene mutation assays } & \multicolumn{2}{|c|}{ Endogenous gene mutation assays } \\
\hline & Whole exome & & Whole exome & & 63 loci (197 Mb, & & gpt (this study) & & $g p t^{2}$ & $\operatorname{lac} z^{3}$ & $\operatorname{lac} Z^{4}$ & $|a c|^{5}$ & Hprt (cDNA) ${ }^{6}$ & Hprt (exon 3) \\
\hline $\begin{array}{l}\text { Target } \\
\text { tissues }\end{array}$ & Offspring & & Offspring & & Offspring & & Sperm & & $\begin{array}{l}\text { Bone } \\
\text { marow }\end{array}$ & Liver & $\begin{array}{l}\text { Bone } \\
\text { marrow }\end{array}$ & $\begin{array}{l}\text { Splenic } \\
\text { lymphocytes }\end{array}$ & $\begin{array}{l}\text { Splenic } \\
\text { lymphocytes }\end{array}$ & $\begin{array}{l}\text { Splenic } \\
\text { lymphocytes }\end{array}$ \\
\hline Mutations & No. & $\%$ & No. & $\%$ & No. & $\%$ & No. & $\%$ & No. \% & No. & No. & No. & No. & No. \\
\hline
\end{tabular}

Base

$\% \quad$ No.

substitution

Transition

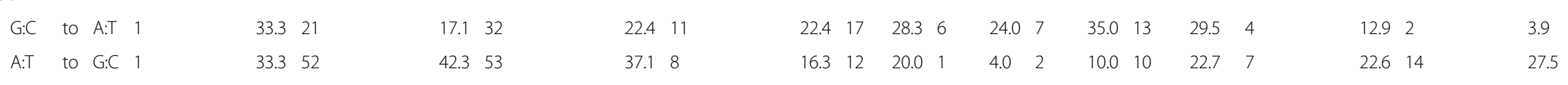

Transversion

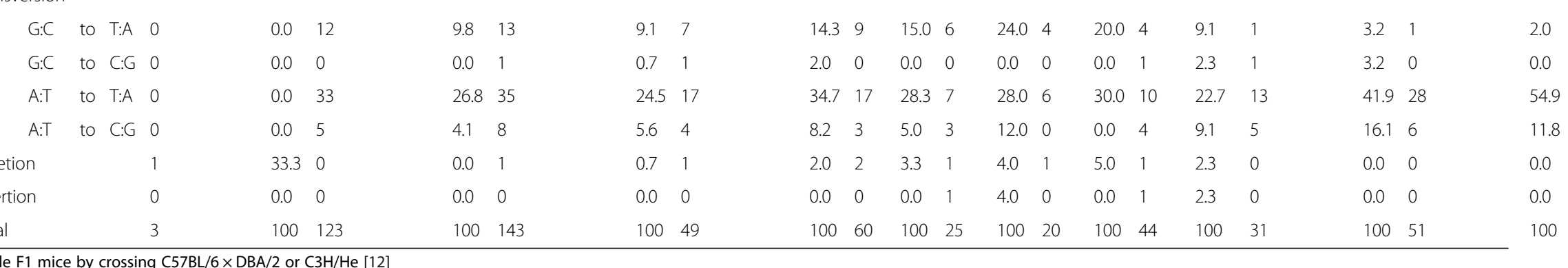

${ }^{1}$ Male F1 mice by crossing C57BL/6 $\times$ DBA/2 or $\mathrm{C} 3 \mathrm{H} / \mathrm{He}[12]$

${ }^{2}$ Male BDF1 gpt delta mice [10]

${ }^{3}$ Female CD2F1 MutaT ${ }^{\mathrm{TM}}$ mice [17]

${ }^{4}$ Male CD2F1 Muta ${ }^{\mathrm{TM}}$ mice [18]

${ }^{5}$ Male B6C3F1 Big Blue ${ }^{\oplus}$ mice [19]

${ }^{6} \mathrm{C} 57 \mathrm{BL} / 6$ mice [30]

${ }^{7}$ Male B6C3F1 mice [19]

${ }^{a}$ Temperature gradient capillary electrophoresis (TGCE) and Sanger sequencing methods 
detected in the ENU and control groups. To avoid underestimating the MF, we calculated the number of nucleotides in the exome, which were sorted by the same condition for mutation detection (read depth $\geq 40$ in all three mice), and the MFs were estimated.

The frequency of confirmed inherited mutations in the ENU-treated father was $184 \pm 48 \times 10^{-8}$ bases (Table 1). The RIKEN ENU mutagenesis project estimated the ENU-induced germline MF as $74.9 \times 10^{-8}$ bases according to a genome-wide screening of $197 \mathrm{Mbp}$ of $7472 \mathrm{~F} 1$ genomes using temperature gradient capillary electrophoresis (TGCE) and Sanger sequencing [12]. Quwailid et al. performed gene-based screening of 27.4 Mbp of 6000 F1 DNA using denaturing high-performance liquid chromatography (HPLC) and determined a mutation rate of 1 in $1 \mathrm{Mbp}$ [15]. Concepcion et al. analyzed a 9.6 $\mathrm{Mb}$ sequence in $510 \mathrm{~F} 1$ mice and estimated a mutation rate of $1.04 \times 10^{-6}$ [16]. These ENU mutagenesis studies were performed using a similar dosing design to our study, i.e., two or three weekly intraperitoneal injections with 75-100 $\mathrm{mg}$ ENU/kg body weight. Therefore, the ENU-induced MF range estimated by NGS in the present study is comparable to those obtained using other detection methods, such as TGCE, HPLC, and direct sequencing.

The mutation spectrum of the ENU-induced inherited mutations is summarized in Table 2. The characteristic ENU-induced mutations comprised base substitutions at A:T bps, where A:T to G:C (42.3\%) and A:T to T:A (26.8 \%) were mainly observed in this study. The mutation spectrum obtained from this NGS-based study appears to be considerably similar to the spectrum produced in another genome-wide analysis using TCGE [12]. However, the predominant type of $g p t$ mutation in the sperm of ENU-treated mice was A:T to T:A (34.7 \%), followed by G:C to A:T (22.4 \%) and A:T to G:C (16.3\%). Transgenic reporter gene assays have demonstrated that the mutation spectra of ENU-induced somatic mutations include base substitutions at both A:T and $\mathrm{G}: \mathrm{C}$ bps $[10,17-19]$, where A:T to T:A and G:C to $\mathrm{A}: \mathrm{T}$ were the predominant mutations in the lacZ, lacI, and gpt genes. This discrepancy may be explained by the different targets used for mutation detection. The TGR mutation assays used reporter genes for E. coli or lambda phage integrated in the mouse chromosome, which were not expressed and highly methylated [20, $21]$. Whole exome sequencing mainly targets exons that are expressed regions in the genome. ENU reacts directly with DNA and generates a variety of DNA adducts $[22,23]$, in which $O^{4}$-ethylthymine, $O^{2}$-ethylthymine, and $O^{6}$-ethylguanine are considered to be responsible for causing A:T to G:C, A:T to T:A, and G:C to A:T mutations [24-28]. Transcription-coupled repair may explain some of the differences in the mutation spectra due to the effective repair of specific adducts in the exome sequence, e.g., nucleotide excision repair plays an important role in the repair of guanine adducts [29]. The mutation spectra were also compared with those of an endogenous reporter gene, $\mathrm{Hprt}$, in splenic lymphocytes (Table 2). Interestingly, the mutation spectra of Hprt showed that A:T base substitutions were most common, as found in the NGS-driven mutation spectrum $[19,30]$. However, the major types were A:T to $\mathrm{T}$ :A in the Hprt locus, but A:T to G:C according to exome sequencing. These differences in the mutation spectra are complex and they do not have simple explanations, e.g., the Hprt and transgenic reporter gene assays involve phenotypic selection. Thus, the mutational characteristics were biased by phenotypic changes in the cells caused by the mutated gene products. In addition, although the mutation spectra of Hprt were analyzed using cDNA and exon 3, whole exome sequencing includes $>20 \%$ out-of-exon sequences, such as intronic, intergenic, and non-coding regions (Additional file 2: Table S3). The sequence contexts also differ among the genetic regions and organisms. Transgenic reporter genes are bacterial genes and their codon usage differs from that in the mouse. Thus, many factors could have contributed to the differences in the mutation spectra. In principle, genotypic selection might reflect in vivo mutagenesis better than phenotypic selection because there is no selection bias. However, it should be noted that NGS also has technical biases in terms of sequencing and mutation detection, such as a preference for point mutations rather than larger indels.

The background control MF $\left(11 \pm 14 \times 10^{-8}\right.$ bases $)$ and mutation spectrum are difficult to discuss because of the high standard deviation and the fact only three mutants were confirmed. The germline mutation rate in humans is considered to be about $1 \times 10^{-8}$ per base per generation [31-35]. The control germline and somatic MFs in mice were also estimated as $1 \times 10^{-8}$ per base or less [36, 37]. In the whole exome sequencing, we used a $49.6 \mathrm{Mb}$ target sequence for each mouse. According to the sorting condition employed in this study, i.e., read depth $\geq 40$ in all three mice, the passed nucleotide sequences in the exome comprised 13.6-13.9 \% in the control group and $26.8-37.5 \%$ in the ENU group (Additional file 3: Figure S2). Therefore, the control MF of each animal could have been zero because no mutations were detected. Thus, more control mice and a deeper read depth may be needed to analyze the background MF. Pooling the background data obtained from the exome sequencing of untreated control mice could also be useful for estimating the background MF and standard deviation in laboratory mice.

The gpt mutant frequency in the sperm of ENUtreated father $\left(44.4 \pm 25.9 \times 10^{-6}\right)$ was 21 times higher 
than that in the control $\left(2.1 \pm 1.7 \times 10^{-6}\right)$. However, the gpt mutant frequency in the liver of the ENUtreated father's offspring was the same as the background level $\left(3.0 \pm 3.6 \times 10^{-6}\right)$. If ENU-induced germline gpt mutations are transmitted to offspring, then a very high $g p t$ mutant frequency could be observed in the F1 mice. It has been reported that there are approximately 40 copies of each transgene per haploid in gpt delta mice [38]. If one copy contains the inherited gpt mutation, then the expected mutant frequency in the $\mathrm{F} 1$ mice would be $1 / 80=12.5 \times 10^{-3}$, which is 1000 times higher than the control level. Based on the 24 F1 mice obtained in this study, we screened $24 \times 40=960$ paternal gpt loci. However, we did not observe clonal mutant mice. The gpt mutant frequency in the sperm from the ENUtreated mice was $44.4 \times 10^{-6}$, and thus we would require 500 offspring to search for 20000 loci to detect germline mutations at a frequency of $50 \times 10^{-6}$. In fact, Barnett et al. treated male Big Blue ${ }^{\mathrm{R}}$ mice with $300 \mathrm{mg}$ ENU/kg body weight and searched for transmitted mutations in 280 offspring, thereby finding four mice with clonal lacI mutations in their whole bodies [39]. In contrast, whole exome sequencing could search approximately $50-\mathrm{Mb}$ regions in each offspring. This suggested that high-throughput DNA sequencing analyses could have higher sensitivity to detect inherited mutations using smaller number of animals.

We compare the germline mutagenesis rates measured by NGS and the gpt assay in Table 3. The frequency of inherited mutations detected by NGS cannot be compared directly with the gpt mutant frequency in sperm, which is based on bacteria-mediated phenotypic selection using a reporter gene, but the increases in the ENU-induced MFs were comparable in both the NGS and gpt assay. This suggests that germ cells from the ENU-treated father could be a good endpoint for evaluating germline mutations, which are origins of inherited mutations. It would be interesting to compare the mutational characteristics of germ cell mutations and inherited mutations, but the differences in the mutation spectra obtained by the two methods may reflect different endpoints, e.g., whether the target sequence is expressed or not. It should be noted that the control MF in this whole exome-based study was higher than expected and it had a high standard deviation, and the problem of false positives still needs to be solved. Additional NGS data based on analyses of more families will help to obtain a more accurate estimation of the MF. In particular, more control mice should be analyzed to estimate the background MF. By using a genetically-closed colony of laboratory animals, the sequence data could be pooled from independent experiments. If the sequencing cost decreases, whole genome sequencing will also be a better solution than whole exome sequencing. In addition, genome-wide instability including copy number and structural variations could also be important targets, as well as point mutations. The detection of de novo mutations by NGS does not need specific transgenic animals with reporter genes and it can be applied to any organism. Thus, this could be a powerful tool for investigating inherited mutations and evaluating the mutagenic effects of chemicals in the next generation in human populations.

\section{Conclusions}

In this study, we analyzed ENU-induced trans-generational mutations by NGS with Sanger sequencing. ENU-treated and control families of gpt delta mice were subjected to whole exome sequencing analyses and we detected de novo mutations in the offspring. The frequency of inherited mutations in the offspring of the ENU-treated family was 17 times higher than that in the control family, and it was comparable to the gpt mutant frequency in the sperm of the ENU-treated mice. The ENU-induced inherited mutation spectrum determined by NGS identified characteristic base substitutions, such as A:T to G:C and A:T to T:A. These results suggest that direct sequencing analyses using

Table 3 Summary of the germlime mutagenicity estimated by NGS and TGR mutation assay

\begin{tabular}{lll}
\hline & $\begin{array}{l}\text { NGS study } \\
\text { (Confirmed by Sanger seq.) }\end{array}$ & TGR mutation assay \\
\hline Background MF & $11 \pm 14 \times 10^{-8} / \mathrm{base}^{\mathrm{a}}$ & $2.1 \pm 1.7 \times 10^{-6} / \mathrm{reporter} \mathrm{gene}^{\mathrm{b}}$ \\
ENU-induced MF & $184 \pm 48 \times 10^{-8} / \mathrm{base}^{\mathrm{a}}$ & $44.4 \pm 25.9 \times 10^{-6} / \mathrm{reporter} \mathrm{gene}^{\mathrm{b}}$ \\
(Fold increase) & $(17$-fold) & $(21$-fold) \\
Target sequence & Whole exome & Neutral transgene \\
& $(49.6 \mathrm{Mb})$ & (gpt: 456 bps) \\
Method of detection & Direct sequencing & Bacteria-mediated phenotypic selection \\
Source of DNA & Liver of offspring & Sperm of treated father \\
& (Inherited mutation) & (Germ cell mutation) \\
\hline
\end{tabular}

Independent mutation frequency. Unit = 1. (Each unit contains father, mother and $4 \mathrm{~F} 1 \mathrm{~s}$.)

${ }^{\mathrm{b}}$ gpt mutant frequency. $\mathrm{N}=5$ 
NGS could be a powerful tool for investigating inherited germline mutations and for evaluating the genotoxic effects of mutagens in the next generation. In addition, germ cells could also be a good endpoint for evaluating germline mutations.

\section{Additional files}

Additional file 1: Figure S1. Design of the animal experiment. Paternal (P), maternal (M), and offspring (F1) mice are shown. (PPTX $79 \mathrm{~kb}$ )

Additional file 2: Table S1. Number of the mutation candidates in the offspring of ENU-treated and vehicle-treated fathers. Table S2. List of the NGS-detected de novo mutations in the offspring of ENU-treated and control father. Table S3. Types of the confirmed de novo mutations detected by whole exome sequencing. (XLSX $40 \mathrm{~kb}$ )

Additional file 3: Figure S2. Percentage of nucleotides in the exome sorted according to the same condition for mutation detection in each of the offspring. Sorting conditions: read depth $\geq 40$ and GQ score $\geq 20$ for each of the three mice (father, mother, and one offspring). (PPTX $187 \mathrm{~kb}$ )

\section{Abbreviations}

6TG: 6-thioguanine; Cm: chloramphenicol; ENU: N-ethyl-N-nitrosourea; Gb: Gigabase; GQ: genotype quality; HPLC: high-performance liquid chromatography; MF: mutation frequency; NGS: next-generation sequencing; SDS: sodium dodecyl sulfate; SNV: single nucleotide variant; SSC: saline sodium citrate; TGCE: temperature gradient capillary electrophoresis; TGR: transgenic rodent.

\section{Competing interests}

The authors declare that they have no competing interests.

\section{Authors' contributions}

KM designed the study, performed the experiment and data analyses, and drafted the manuscript. NT performed the animal experiment and mutation assay. AU performed the mutation assay. YG helped design the experiment. $\mathrm{MH}$ helped to draft the manuscript. TN conceived the study, participated in its design and coordination, and helped to draft the manuscript. All of the authors read and approved the final manuscript.

\section{Acknowledgments}

This study was supported by JSPS KAKENHI Grant Number 25281027 for KM, 25241016 for $Y G$.

\section{Author details}

${ }^{1}$ Division of Genetics and Mutagenesis, National Institute of Health Sciences, 1-18-1 Kamiyoga, Setagaya-ku, Tokyo 158-8501, Japan. ${ }^{2}$ RIKEN BioResource Center, 3-1-1 Koyadai, Tsukuba, Ibaraki 305-0074, Japan. ${ }^{3}$ Biological Safety Research Center, National Institute of Health Sciences, 1-18-1 Kamiyoga, Setagaya-ku, Tokyo 158-8501, Japan.

Received: 23 December 2015 Accepted: 11 February 2016 Published online: 01 April 2016

\section{References}

1. Yauk CL, Aardema MJ, Benthem J, Bishop JB, Dearfield KL, DeMarini DM, et al. Approaches for identifying germ cell mutagens: Report of the 2013 IWGT workshop on germ cell assays. Mutat Res. 2015;783:36-54.

2. Demarini DM. Declaring the existence of human germ-cell mutagens. Environ Mol Mutagen. 2012;53:166-72.

3. OECD. Test No. 485: Genetic toxicology, Mouse Heritable Translocation Assay, OECD Guidelines for the Testing of Chemicals, Section 4. 1986.

4. Davis AP, Justice MJ. An Oak Ridge legacy: the specific locus test and its role in mouse mutagenesis. Genetics. 1998;148:7-12.

5. Lambert IB, Singer TM, Boucher SE, Douglas GR. Detailed review of transgenic rodent mutation assays. Mutat Res. 2005;590:1-280.
6. Justice MJ, Noveroske JK, Weber JS, Zheng B, Bradley A. Mouse ENU mutagenesis. Hum Mol Genet. 1999:8:1955-63.

7. Hrabe de Angelis M, Balling R. Large scale ENU screens in the mouse: genetics meets genomics. Mutat Res. 1998;400:25-32.

8. Inoue M, Sakuraba Y, Motegi H, Kubota N, Toki H, Matsui J, et al. A series of maturity onset diabetes of the young, type 2 (MODY2) mouse models generated by a large-scale ENU mutagenesis program. Hum Mol Genet. 2004;13:1147-57.

9. Nohmi T, Katoh M, Suzuki H, Matsui M, Yamada M, Watanabe M, et al. A new transgenic mouse mutagenesis test system using Spi ${ }^{-}$and 6thioguanine selections. Environ Mol Mutagen. 1996;28:465-70.

10. Masumura K, Matsui M, Katoh M, Horiya N, Ueda O, Tanabe H, et al. Spectra of gpt mutations in ethylnitrosourea-treated and untreated transgenic mice. Environ Mol Mutagen. 1999;34:1-8.

11. Justice MJ. Mouse Mutagenesis Using N-Ethyl-N-Nitrosourea (ENU). Cold Spring Harb Protoc. 2008. doi:10.1101/pdb.prot4985.

12. Sakuraba Y, Sezutsu H, Takahasi KR, Tsuchihashi K, Ichikawa R, Fujimoto N, et al. Molecular characterization of ENU mouse mutagenesis and archives. Biochem Biophys Res Commun. 2005:336:609-16.

13. Yauk $C L$, Dubrova $Y E$, Grant GR, Jeffreys AJ. A novel single molecule analysis of spontaneous and radiation-induced mutation at a mouse tandem repeat locus. Mutat Res. 2002;500:147-56.

14. Nohmi T, Suzuki T, Masumura K. Recent advances in the protocols of transgenic mouse mutation assays. Mutat Res. 2000;455:191-215.

15. Quwailid MM, Hugill A, Dear N, Vizor L, Wells S, Horner E, et al. A genedriven ENU-based approach to generating an allelic series in any gene. Mamm Genome. 2004;15:585-91.

16. Concepcion D, Seburn KL, Wen G, Frankel WN, Hamilton BA. Mutation rate and predicted phenotypic target sizes in ethylnitrosourea-treated mice. Genetics. 2004;168:953-9.

17. Mientjes EJ, Luiten-Schuite A, van der Wolf E, Borsboom Y, Bergmans A, Berends F, et al. DNA adducts, mutant frequencies, and mutation spectra in various organs of lambda lacZ mice exposed to ethylating agents. Environ Mol Mutagen. 1998;31:18-31.

18. Suzuki T, Hayashi M, Wang X, Yamamoto K, Ono T, Myhr BC, et al. A comparison of the genotoxicity of ethylnitrosourea and ethyl methanesulfonate in lacZ transgenic mice (Muta ${ }^{\left({ }^{M}\right)}$ Mouse). Mutat Res. 1997;395:75-82.

19. Walker VE, Gorelick NJ, Andrews JL, Craft TR, deBoer JG, Glickman BW, et al. Frequency and spectrum of ethylnitrosourea-induced mutation at the hprt and lacl loci in splenic lymphocytes of exposed lacl transgenic mice. Cancer Res. 1996;56:4654-61.

20. Gossen JA, de Leeuw WJ, Tan CH, Zwarthoff EC, Berends F, Lohman PH, et al. Efficient rescue of integrated shuttle vectors from transgenic mice: a model for studying mutations in vivo. Proc Natl Acad Sci U S A. 1989:86:7971-5

21. Heddle JA, Martus HJ, Douglas GR. Treatment and sampling protocols for transgenic mutation assays. Environ Mol Mutagen. 2003;41:1-6.

22. Shibuya T, Morimoto K. A review of the genotoxicity of 1-ethyl-1nitrosourea. Mutat Res. 1993;297:3-38.

23. Zielenska M, Beranek D, Guttenplan JB. Different mutational profiles induced by $\mathrm{N}$-nitroso-N-ethylurea: effects of dose and error-prone DNA repair and correlations with DNA adducts. Environ Mol Mutagen. 1988;11:473-85.

24. Singer B, Dosanjh MK. Site-directed mutagenesis for quantitation of basebase interactions at defined sites. Mutat Res. 1990;233:45-51.

25. Beranek DT. Distribution of methyl and ethyl adducts following alkylation with monofunctional alkylating agents. Mutat Res. 1990;231:11-30.

26. Klein JC, Bleeker MJ, Lutgerink JT, van-Dijk WJ, Brugghe HF, van-den-Elst $H$, et al. Use of shuttle vectors to study the molecular processing of defined carcinogen-induced DNA damage: mutagenicity of single $0^{4}$-ethylthymine adducts in HeLa cells. Nucleic Acids Res. 1990;18:4131-7.

27. Bhanot OS, Grevatt PC, Donahue JM, Gabrielides CN, Solomon JJ. In vitro DNA replication implicates $\mathrm{O}^{2}$-ethyldeoxythymidine in transversion mutagenesis by ethylating agents. Nucleic Acids Res. 1992;20:587-94.

28. Singer B, Chavez F, Goodman MF, Essigmann JM, Dosanjh MK. Effect of 3' flanking neighbors on kinetics of pairing of dCTP or dTTP opposite $O^{6}$ methylguanine in a defined primed oligonucleotide when Escherichia coli DNA polymerase I is used. Proc Natl Acad Sci U S A. 1989;86:8271-4.

29. Bronstein SM, Skopek TR, Swenberg JA. Efficient repair of $O^{6}$-ethylguanine, but not $O^{4}$-ethylthymine or $O^{2}$-ethylthymine, is dependent upon $O^{6}$ alkylguanine-DNA alkyltransferase and nucleotide excision repair activities in human cells. Cancer Res. 1992;52:2008-11. 
30. Dobrovolsky VN, Chen T, Heflich RH. Molecular analysis of in vivo mutations induced by $\mathrm{N}$-ethyl- $\mathrm{N}$-nitrosourea in the autosomal Tk and the $\mathrm{X}$-linked Hprt genes of mouse lymphocytes. Environ Mol Mutagen. 1999;34:30-8.

31. Shendure J, Akey JM. The origins, determinants, and consequences of human mutations. Science. 2015;349:1478-83.

32. Conrad DF, Keebler JE, DePristo MA, Lindsay SJ, Zhang Y, Casals F, et al. Variation in genome-wide mutation rates within and between human families. Nat Genet. 2011;43:712-4.

33. Kong A, Frigge ML, Masson G, Besenbacher S, Sulem P, Magnusson G, et al. Rate of de novo mutations and the importance of father's age to disease risk. Nature. 2012:488:471-5.

34. Lynch M. Rate, molecular spectrum, and consequences of human mutation. Proc Natl Acad Sci U S A. 2010;107:961-8.

35. Nachman MW, Crowell SL. Estimate of the mutation rate per nucleotide in humans. Genetics. 2000;156:297-304.

36. Uchimura A, Higuchi M, Minakuchi Y, Ohno M, Toyoda A, Fujiyama A, et al. Germline mutation rates and the long-term phenotypic effects of mutation accumulation in wild-type laboratory mice and mutator mice. Genome Res. 2015;25:1125-34

37. Wright $J \mathrm{H}$, Modjeski KL, Bielas JH, Preston BD, Fausto N, Loeb LA, et al. A random mutation capture assay to detect genomic point mutations in mouse tissue. Nucleic Acids Res. 2011:39:e73.

38. Masumura K, Sakamoto Y, Kumita W, Honma M, Nishikawa A, Nohmi T. Genomic integration of lambda EG10 transgene in gpt delta transgenic rodents. Genes and Environment. 2015;37:24.

39. Barnett LB, Tyl RW, Shane BS, Shelby MD, Lewis SE. Transmission of mutations in the lacl transgene to the offspring of ENU-treated Big Blue male mice. Environ Mol Mutagen. 2002:40:251-7.

\section{Submit your next manuscript to BioMed Central and we will help you at every step:}

- We accept pre-submission inquiries

- Our selector tool helps you to find the most relevant journal

- We provide round the clock customer support

- Convenient online submission

- Thorough peer review

- Inclusion in PubMed and all major indexing services

- Maximum visibility for your research

Submit your manuscript at www.biomedcentral.com/submit 